\title{
HOW TO REPRESENT THE GENETIC CODE?
}

\author{
Santos-Magalhães, N.S. ${ }^{1}$; Bouton, E.A. ${ }^{2}$; de Oliveira, H.M. ${ }^{2}$ \\ Universidade Federal de Pernambuco \\ ${ }^{1}$ Depto de Bioquímica-Lab. de Imunologia Keiso-Asami LIKA, ${ }^{2}$ Depto de Eletrônica e Sistemas
}

The advent of molecular genetic comprises a true revolution of far-reaching consequences for humankind, which evolved into a specialized branch of the modern-day Biochemistry. The analysis of specific genomic information are gaining wide-ranging interest because of their significance to the early diagnosis of disease, and the discovery of modern drugs. In order to take advantage of a wide assortment of signal processing (SP) algorithms, the primary step of modern genomic SP involves converting symbolic-DNA sequences into complex-valued signals. How to represent the genetic code? Despite being extensively known, the DNA mapping into proteins is one of the relevant discoveries of genetics. The genetic code (GC) is revisited in this work, addressing other descriptions for it, which can be worthy for genomic SP. Three original representations are discussed. The inner-to-outer map builds on the unbalanced role of nucleotides of a codon. A two-dimensional-Gray genetic representation is offered as a structured map that can help interpreting DNA spectrograms or scalograms. These are among the powerful visual tools for genome analysis, which depends on the choice of the genetic mapping. Finally, the world-chart for the GC is investigated. Evoking the cyclic structure of the genetic mapping, it can be folded joining the left-right borders, and the top-bottom frontiers. As a result, the GC can be drawn on the surface of a sphere resembling a world-map. Eight parallels of latitude are required (four in each hemisphere) as well as four meridians of longitude associated to four corresponding anti-meridians. The tropic circles have $11.25^{\circ}, 33.75^{\circ}, 56.25^{\circ}$, and $78.5^{\circ}$ (North and South). Starting from an arbitrary Greenwich meridian, the meridians of longitude can be plotted at $22.5^{\circ}, 67.5^{\circ}, 112.5^{\circ}$, and $157.5^{\circ}$ (East and West). Each triplet is assigned to a single point on the surface that we named Nirenberg-Kohama's Earth. Despite being valuable, usual representations for the GC can be replaced by the handy descriptions offered in this work. These alternative maps are also particularly useful for educational purposes, giving a much rich interpretation and visualization than a simple look-up table. 